\title{
Circumferential esophageal perforation resulting in tension hydropneumothorax in a patient with septic shock
}

\author{
Saad Saffo', James Farrell', Anil Nagar ${ }^{1,2}$ \\ ${ }^{1}$ Department of Internal Medicine, Section of Digestive Diseases, Yale University School of Medicine, New Haven, CT; ${ }^{2}$ West Haven Veteran Affairs Medical \\ Center, West Haven, CT, USA
}

Esophageal perforations occur traumatically or spontaneously and are typically associated with high mortality rates. Early recognition and prompt management are essential. We present the case of a 76-year-old man who was admitted to the medical intensive care unit with fulminant Clostridium difficile colitis, shock, and multi-organ failure. After an initial period of improvement, his condition rapidly deteriorated despite aggressive medical management, and he required mechanical ventilation. Radiography after endotracheal intubation showed interval development of pneumomediastinum and bilateral hydropneumothorax with tension physiology. Chest tube placement resulted in the drainage of multiple liters of dark fluid, and pleural fluid analysis was notable for polymicrobial empyemas. Despite the unusual presentation, esophageal perforation was suspected. Endoscopy ultimately confirmed circumferential separation of the distal esophagus from the stomach, and bedside endoscopic stenting was performed with transient improvement. Two weeks after admission, he developed mediastinitis complicated by recurrent respiratory failure and passed away. This report further characterizes our patient's unique presentation and briefly highlights the clinical manifestations, management options, and outcomes of esophageal perforations.

Key Words: esophageal perforation; hydropneumothorax; septic shock

Esophageal perforations can occur after various types of chemical or mechanical trauma, as a postoperative complication, in the setting of malignancy, or spontaneously. Referred to as Boerhaave syndrome, spontaneous perforation occurs when intraesophageal pressure rises in the face of low intrathoracic pressure, most commonly due to forceful retching and vomiting. Most perforations occur in the distal intrathoracic or abdominal esophagus, and a timely diagnosis relies on a high index of clinical suspicion, along with confirmatory laboratory, radiographic, and/or endoscopic findings [1,2].

Immediate management options include close medical observation or surgical and endoscopic therapies to either repair the defect or prevent further extravasation of luminal contents into the mediastinum or pleural spaces. The type of intervention typically depends on the patient's clinical stability, the size and location of the lesion, and a variety of other host factors. If appropriate medical and surgical interventions are performed within the first 24 hours, the 30 -day in-hospital mortality rate is below $8 \%$, but with delays, the mortality rate

\section{Case Report}

Received: December 1, 2020

Revised: January 6, 2021

Accepted: February 15, 2021

Corresponding author

Saad Saffo

Department of Internal Medicine, Section of Digestive Diseases, Yale University School of Medicine, 333 Cedar St, 1080 LMP, New Haven, CT 06520-8019, USA

Tel: +1-2039883907

Fax: +1-9842038299

E-mail: saad.saffo@yale.edu

Copyright $(\odot 2021$ The Korean Society of Critical Care Medicine

This is an Open Access article distributed under the terms of Creative Attributions Non-Commercial License (https:// creativecommons.org/li-censes/by-nc/4.0/) which permits unrestricted noncommercial use, distribution, and reproduction in any medium, provided the original work is properly cited. 
rises above $20 \%$ [1-3]. Stratified by location, abdominal perforations carry the highest mortality rate, exceeding $13 \%$ [1]. Patients who undergo endoscopic interventions may require reintervention, including surgery, though there is no clear difference in mortality among treatment strategies in most instances $[1,2,4]$.

We present an atypical case of esophageal perforation in a critically-ill patient. Our aims are to (1) highlight unique aspects of his clinical presentation, (2) demonstrate the importance of considering acute esophageal pathology among decompensating patients in the intensive care unit (ICU), and (3) describe the evolution of management strategies and outcomes for esophageal perforations.

\section{CASE REPORT}

A frail 76-year-old man with a history of bladder cancer with regional lymph node involvement and Clostridium difficile infection (CDI) presented to the hospital from his skilled nursing facility with several days of vomiting, watery diarrhea, and anorexia in the context of mild odynophagia. Prior to his admission, diagnostic work-up confirmed recurrent CDI, and he had been started on oral vancomycin with minimal symptomatic improvement.

On arrival to the emergency department, the patient was alert and interactive but hypotensive with a blood pressure of 80/40 mm Hg. Labs revealed acute renal dysfunction, lactic acidosis, and leukocytosis with bandemia. Plain films of the chest showed basilar atelectasis (Figure 1A), and computed tomography (CT) of the abdomen and pelvis was notable for diffuse colonic wall-thickening and peri-colonic fat-stranding without bowel dilatation or perforation. He received sequential boluses of $2 \mathrm{~L}$ of normal saline and $3 \mathrm{~L}$ of lactated ringers over a period of 4 hours, norepinephrine titrated to a rate of $0.4 \mu \mathrm{g} / \mathrm{kg} / \mathrm{min}$, and oral vancomycin plus broad-spectrum intravenous antibiotics, with subsequent clinical improvement. He was admitted to the medical ICU for further management of septic shock secondary to fulminant CDI. At that time, mental status was excellent, urine output was in excess of 0.5 $\mathrm{mL} / \mathrm{kg} / \mathrm{hr}$, and repeat lab work revealed resolving lactic acidosis.

However, approximately 6 hours later, he developed unexplained back pain and briskly deteriorated with worsening shock and multi-organ failure requiring five vasopressors. Plain films of the abdomen were unrevealing. Emergent endotracheal intubation with orogastric tube placement was performed. A post-procedure chest X-ray revealed bilateral hydropneumothorax and pneumomediastinum (Figure 1B), and placement of bilateral chest tubes yielded more than $1 \mathrm{~L}$ of dark fluid from each pleural space (Figure 1C). Esophageal perforation was suspected, but in the context of clinical instability, cross-sectional imaging was initially deferred. Pleural fluid analysis was notable for a markedly elevated amylase level of 2,238 units/L (serum amylase was 84 units/L; reference range, 9-136 units/L), and Gram stain revealed Grampositive cocci and rods. Pleural cultures grew multiple species of aerobic Gram-positive organisms.

In the context of shock complicated by progressive renal impairment, he subsequently developed a mixed refractory acidemia. Continuous veno-venous hemodiafiltration was
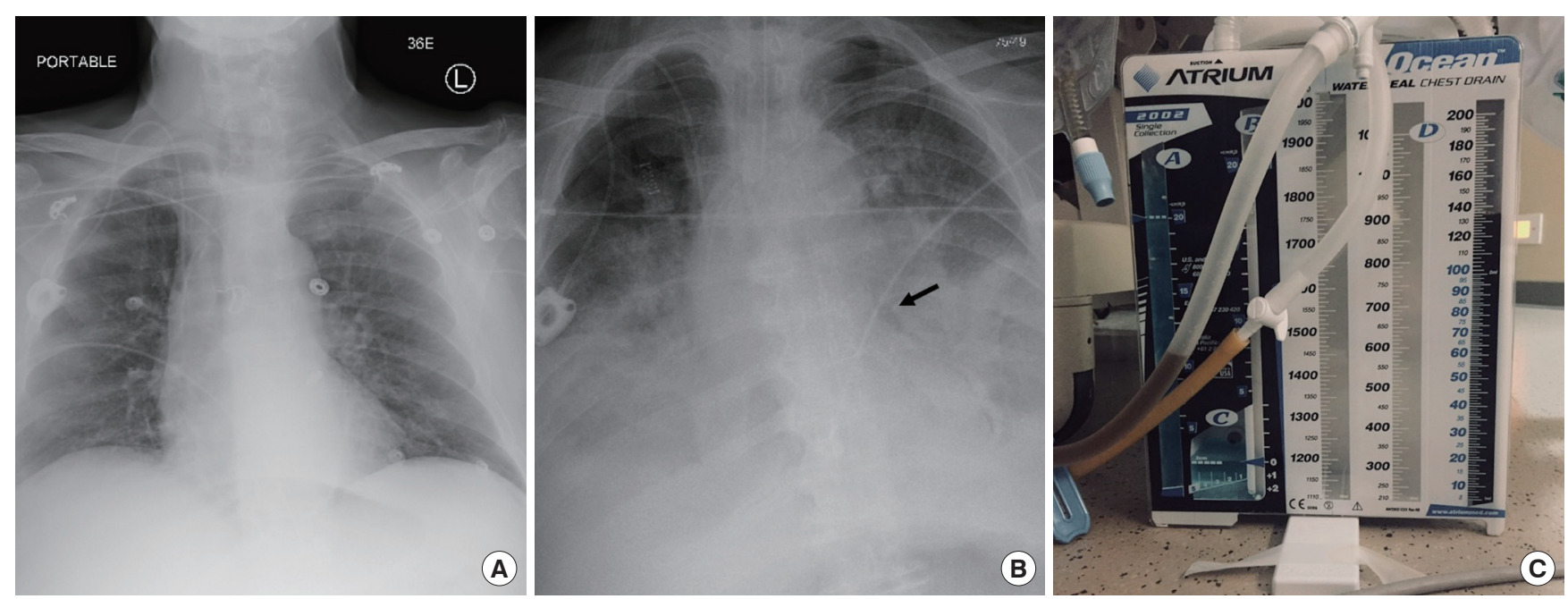

Figure 1. Plain films of the chest on presentation (A) and after (B) endotracheal intubation revealing development of bilateral hydropneumothorax and pneumomediastinum (black arrow). After chest tube placement, dark fluid was evacuated (C). 

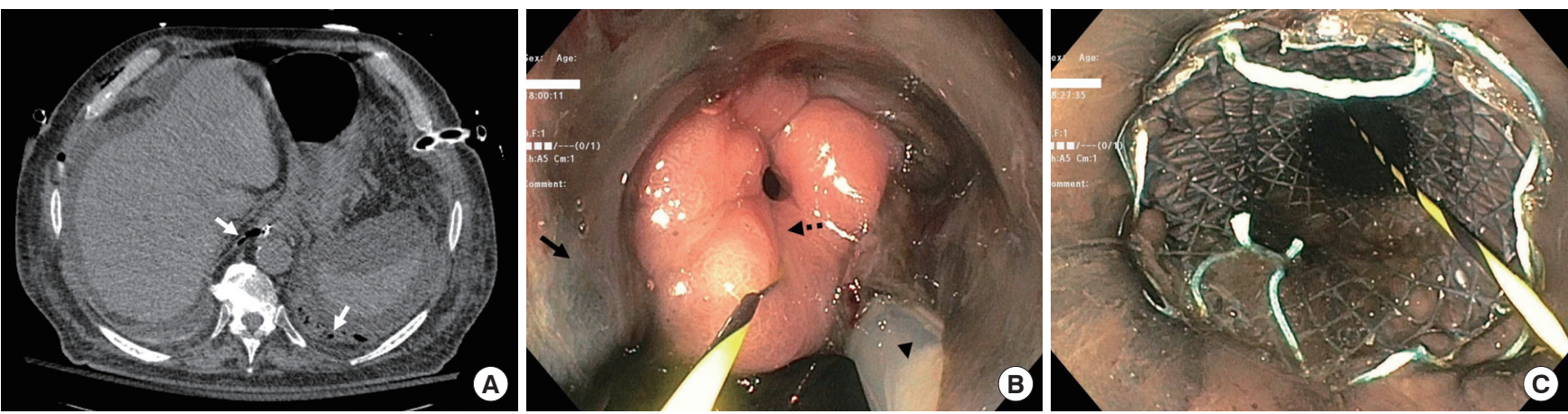

Figure 2. Computed tomography (A) revealing air tracking from the esophagus to the pleural spaces (solid white arrows) and endoscopy (B) revealing separation of the necrotic esophagus (solid black arrow) from viable stomach (dashed black arrow) with a nasogastric tube (arrowhead) going through the perforation into the mediastinum. An unsutured fully-covered metal stent was deployed across the perforation (C).

initiated, and with ongoing mechanical ventilation and vasopressor support, his condition stabilized. Approximately 24 hours later, CT of the chest was performed and highly suggestive of esophageal perforation with locules of air communicating from the pleural spaces to the distal esophagus (Figure 2A) and an orogastric tube penetrating across the perforation into the lesser sac of the stomach.

After multi-disciplinary discussions involving the critical care, thoracic surgery, and gastroenterology teams and the patient's family, endoscopic intervention was performed at bedside on hospital day 3 without the use of fluoroscopy, revealing extensive esophageal necrosis and complete separation of the lower esophagus from the stomach (Figure 2B). An unsutured fully-covered self-expanding $18 \mathrm{~mm} \times 15.3 \mathrm{~cm}$ metal stent (WallFlex; Boston Scientific, Marlborough, MA, USA) was deployed using an over-the-guidewire approach along the gastroesophageal junction, between 32 and $47 \mathrm{~cm}$ from the incisors, traversing the regions of necrosis and perforation (Figure 2C). The patient was started on parental nutrition, and chest tubes were maintained. By hospital day 7, vasopressors were titrated off, dialysis was discontinued, and he was extubated. Surgical intervention was deferred. Unfortunately, 1 week later, he developed mediastinitis complicated by recurrent respiratory failure. The patient and his family chose to pursue a comfort-focused approach, and he passed away without further interventions.

\section{DISCUSSION}

Our patient presented with fulminant colitis and septic shock secondary to CDI and initially improved after receiving appropriate medical therapy. His subsequent clinical deterioration was suggestive of a new process, which proved to be an esophageal perforation complicated by mixed shock second- ary to tension hydropneumothorax and sepsis. However, the cause of the perforation was debatable. Although he had preceding vomiting raising suspicion for Boerhaave syndrome, there are clues which suggest that the initial episode of shock may have been a critical contributing factor. First, in light of the patient's marked frailty, it is unlikely that the prior episodes of vomiting could have generated intraesophageal pressure amplitudes sufficient to cause spontaneous perforation. Second, his initial chest X-ray and CT scan of the abdomen were not consistent with perforation, and he did not have further episodes of retching or vomiting in the hospital. The more likely explanation is that the patient had erosive esophagitis, and compounded by the presence of shock, this resulted in the development of ischemia, necrosis, and perforation of the esophagus. Endotracheal intubation, including the transient use of bag-valve ventilation and orogastric tube placement likely led to detachment of the necrotic esophageal and gastric compartments.

The patient's management strategy proved to be challenging. Historically, primary surgical repair has been the preferred treatment modality in the majority of instances, although endoscopic interventions are now being utilized more routinely. Because our patient's clinical condition precluded him from surgery, he underwent endoscopic stenting, but based on the magnitude and depth of the perforation, a favorable outcome was unlikely. While there is increasing experience supporting a primary role for endoscopic management alone, the topic remains controversial [5].

Although no prospective randomized controlled trials exist, numerous small retrospective studies have been published with variable findings. A systematic review of more than 700 patients, including 116 with spontaneous perforations, demonstrated that the success rate of self-expandable metal stents was comparable to that of surgical repair with an overall suc- 
cess rate of more than $80 \%$ in both groups and an in-hospital mortality ranging between $7.5 \%$ and $17 \%$ [6]. Another review of 27 case series that included more than 300 patients also showed that the success rate exceeded $80 \%$ and that use of metal rather than plastic stents was associated with a significant reduction in the rate of reintervention [7]. Alternatively, there are a number of studies that suggest that endoscopic stenting is rarely successful on its own, and patients often require reintervention to manage complications such as mediastinitis or empyema. In a small international study among British and German centers, $85 \%$ of patient undergoing endoscopic repair ultimately required surgical intervention whereas, among the patients who underwent surgical repair, $30 \%$ required reintervention [4]. These findings are consistent with data from other recent single-center studies $[8,9]$. Interestingly, amongst most studies, the rates of sepsis and overall mortality are similar with no definite trend favoring one group suggesting that endoscopic stenting retains an important role in the management of critically ill patients, either as a bridge to surgery or a primary modality in select cases. However, it is important to note that the patients included in these studies were unlikely to have complete transection of the lower esophagus. In cases where there is transection or the magnitude of the defect is large and an individual is a candidate for surgery, stenting should likely only be utilized as a bridge to definitive surgical repair.

In summary, we present an unusual case of esophageal perforation in a critically ill patient and highlight the importance of including acute esophageal pathology in the differential for a decompensating host in the ICU. Although tension hydropneumothorax has been previously reported as a rare complication of esophageal perforation due to Boerhaave syndrome, these entities have not been previously described in relation to septic shock [10]. Our review of the literature suggests that intervention within the first 24 hours leads to improved survival. However, studies have not convincingly shown differences in mortality between surgical or endoscopic therapies. Decisions should be patient-centered and rely on institutional expertise and host and disease factors. In this case, the likelihood of achieving a successful outcome with endoscopic stenting alone was low given the size of the patient's perforation and the presence of extensive necrosis. Based on his frailty, comorbidities, and personal preference for nonsurgical management, operative repair was deferred. Unfortunately, he ultimately succumbed to recurrent infections and respiratory failure.

\section{CONFLICT OF INTEREST}

No potential conflict of interest relevant to this article was reported.

\section{ORCID}

Saad Saffo https://orcid.org/0000-0001-5375-3100

James Farrell

Anil Nagar https://orcid.org/0000-0003-4860-5693 https://orcid.org/0000-0003-4946-209X

\section{AUTHOR CONTRIBUTIONS}

Conceptualization: all authors. Data curation, Formal analysis, Methodology, Project administration, Visualization, \& Writing-original draft: SS. Writing-review \& editing: all authors.

\section{REFERENCES}

1. Biancari F, D’Andrea V, Paone R, Di Marco C, Savino G, Koivukangas V, et al. Current treatment and outcome of esophageal perforations in adults: systematic review and meta-analysis of 75 studies. World J Surg 2013;37:1051-9.

2. Puerta Vicente A, Priego Jiménez P, Cornejo López MÁ, García-Moreno Nisa F, Rodríguez Velasco G, Galindo Álvarez J, et al. Management of esophageal perforation: 28-year experience in a major referral center. Am Surg 2018;84:684-9.

3. Muir AD, White J, McGuigan JA, McManus KG, Graham AN Treatment and outcomes of oesophageal perforation in a tertiary referral centre. Eur J Cardiothorac Surg 2003;23:799-804.

4. Schweigert M, Beattie R, Solymosi N, Booth K, Dubecz A Muir A, et al. Endoscopic stent insertion versus primary operative management for spontaneous rupture of the esophagus (Boerhaave syndrome): an international study comparing the outcome. Am Surg 2013;79:634-40.

5. Kopelman Y, Abu baker F, Troiza A, Hebron D. Boerhaave syndrome in an elderly man successfully treated with 3-month indwelling esophageal stent. Radiol Case Rep 2018;13:1084-6.

6. Persson S, Rouvelas I, Irino T, Lundell L. Outcomes following the main treatment options in patients with a leaking esophagus: a systematic literature review. Dis Esophagus 2017;30:110 .

7. Dasari BV, Neely D, Kennedy A, Spence G, Rice P, Mackle E, et al. The role of esophageal stents in the management of esophageal anastomotic leaks and benign esophageal perforations. Ann Surg 2014;259:852-60.

8. Glatz T, Marjanovic G, Kulemann B, Hipp J, Theodor Hopt U, 
Fischer A, et al. Management and outcome of esophageal stenting for spontaneous esophageal perforations. Dis Esophagus 2017;30:1-6.

9. Hauge T, Kleven OC, Johnson E, Hofstad B, Johannessen HO. Outcome after iatrogenic esophageal perforation. Scand J
Gastroenterol 2019;54:140-4.

10. Lieu MT, Layoun ME, Dai D, Soo Hoo GW, Betancourt J. Tension hydropneumothorax as the initial presentation of Boerhaave syndrome. Respir Med Case Rep 2018;25:100-3. 